\title{
Direct Observation of Chain Lengths and Conformations in Oligofluorene Distributions from Controlled Polymerization by Double Electron-Electron Resonance
}

\author{
Dennis Bücker ${ }^{\ddagger a}$, Annika Sickinger ${ }^{\ddagger a}$, Julian D. Ruiz Perez ${ }^{a}$, Manuel Oestringer ${ }^{\text {a }}$, Stefan Mecking ${ }^{\text {a* }}$ and \\ Malte Drescher ${ }^{\text {a* }}$

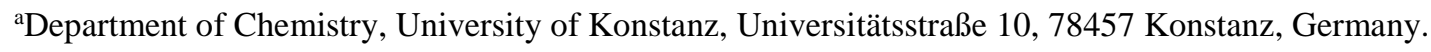

KEYWORDS. Electron paramagnetic resonance, conjugated polymers, controlled polymerization, polymer particles, wormlike chain model, distance determination, double-Electron-Electron resonance (DEER).

\begin{abstract}
Synthetic polymers are mixtures of different length chains, and their chain length and chain conformation is often experimentally characterized by ensemble averages. We demonstrate that Double-Electron-Electron-Resonance (DEER) spectroscopy can reveal the chain length distribution, and chain conformation and flexibility of the individual n-mers in oligo(9,9-dioctylfluorene) from controlled Suzuki-Miyaura Coupling Polymerization (cSMCP). The required spin-labeled chain ends were introduced efficiently via a TEMPO-substituted initiator and chain terminating agent, respectively, with an in situ catalyst system. Individual precise chain length oligomers as reference materials were obtained by a stepwise approach. Chain length distribution, chain conformation and flexibility can also be accessed within poly(fluorene) nanoparticles.
\end{abstract}

\section{Introduction}

Knowledge of the chain length distribution and of chain conformations are essential to understand and design synthetic procedures, materials properties, and nanoscale structures. ${ }^{1,2}$ Consequently numerous methods to access these parameters have been developed to a high level and for practical use. The most prominent and ubiquitious method to access molecular weight distributions is size exclusion chromatography. More recently, mass spectrometry has also been advanced considerably for the lower molecular weight regime, though it is not quantitative. These methods are based on separation of the sample, prior to detection of its individual chain length components. Likewise, an experimental determination of chain conformations on mixtures of different length chains yields an ensemble average only.

We now report double-electron-electron-resonance $(\mathrm{DEER})^{3,4}$ studies of spin-labeled oligofluorene mixtures that give direct access to the chain length distributions and monitor conformational ensembles and flexibilities ${ }^{5}$ of the oligomers.

As a probe we chose oligofluorenes from controlled $\mathrm{Su}$ zuki-Miyaura coupling polymerization (cSMCP) ${ }^{6,7}$ Oligofluorenes are attractive materials due to their photo- and electroluminescence and light-induced charge generation. ${ }^{8,9}$ cSMCP proceeds in a chain growth fashion. ${ }^{10}$ The controlled character of cSMCP allows for an introduction of functional endgroups at both chain ends. ${ }^{10-12} \mathrm{cSMCP}$ has been demonstrated for the synthesis of a scope of polyarylenes, like poly(fluorenes), poly(thiophenes) and poly(phenylenes). ${ }^{7,13}$

DEER is a pulsed electron paramagnetic resonance (EPR) method to determine distance distributions between paramagnetic centers. Nitroxide groups, such as 2,2,6,6-tetramethylpiperidinyloxyl (TEMPO) are commonly used as spin labels. DEER is established in biological chemistry where it is commonly used to determine distances between a pair of spins placed in a single defined type of molecule, like a protein. ${ }^{14,15}$ In the same way, DEER has also been applied to defined monodisperse synthetic molecules. ${ }^{16,17}$

\section{Results and Discussion}

Spin labels were attached at the chain ends of poly $(9,9-$ dioctylfluorene) (PF8) directly during polymerization by employing spin-labeled initiators and terminating agents in an otherwise established controlled SMCP protocol. ${ }^{10,11,18}$ The polymerization was initiated by an in situ system using chloro[(tri-tert-butylphosphine)-2-(amino-biphenyl)] palladium(II) as Pd(0) source, and TEMPO-labeled 4-bromobenzoic acid which adds oxidatively. Chains were quenched by addition of TEMPO-labeled 4-carboxyphenyl-boronic acid pinacol ester end capping agent, resulting in identical chain termini (Figure 1). The successful incorporation of the TEMPO labels was confirmed by MALDI-TOF MS, identi- 
fying the doubly labeled polymer chains as major component by the isotopic pattern (cf. Figure S 4.1). As expected, the maximum of the obtained chain length distribution determined by GPC could be adjusted by the ratio of initiator to monomer in the reaction mixture. Thus, the described in situ system offers a robust and versatile method for the synthesis of doubly TEMPO labeled conjugated polymers in a one-step approach.
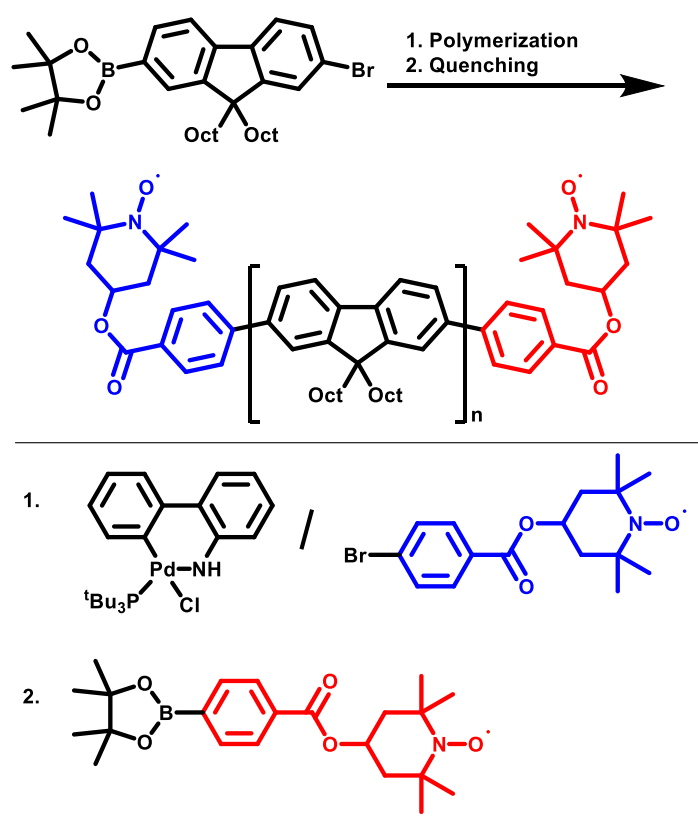

Figure 1. Synthetic approach to double TEMPO-labeled oligo- and polyfluorenes by controlled Suzuki-Miyaura coupling polymerization.

For establishing a chain conformation analysis, doubly labeled monodisperse oligomers with a precise number of repeat units (herein referred to as doubly labeled precise oligomers) were required as a reference. According to reported procedures, the non-labeled oligo(fluorenes) were built up stepwise by repetitive cycles of alternating Suzuki-Miyaura coupling and bromination, starting from the mono- and dibromo-substituted fluorene monomers (Figure S 3.2). ${ }^{19-21}$ Spin labels were attached to the oligomers by a final SuzukiMiyaura coupling step with a TEMPO-substituted monofunctional arylboronic acid ester. These doubly labeled precise oligomers from stepwise synthesis are abbreviated as $\mathbf{P}_{\mathbf{n}}$, with $\mathrm{n}$ representing the chain length in the following. Oligomers up to $n=5$ were synthesized. As the synthetic effort of the step-by-step approach considerably increases for each additional monomer unit, reference oligomers with $\mathrm{n}=5$ and 6, abbreviated as $\mathbf{P}^{\mathbf{5}^{\prime}}$ and $\mathbf{P}_{\mathbf{6}}{ }^{\prime}$, were provided by semi-preparative GPC fractionation (cf. S 3.2).

As reference, DEER measurements in Q-band for the precise oligomers were performed upon shock-freezing in toluene- $\mathrm{d}_{8}$ (Figure $2 \mathrm{~A}$ ). The experimental data for all individual oligomers $\mathbf{P}_{\mathbf{n}}$ (with $\mathrm{n}=1-4$ ), $\mathbf{P}_{5}{ }^{\prime}$ and $\mathbf{P}_{6}{ }^{\prime}$ was fitted (Figure 2A) with the worm-like chain model (WLC), ${ }^{17,22,23}$ using DEERAnalysis. ${ }^{24-26}$ Assuming that the monomer $\mathbf{P}_{1}$ with just one repeat unit is completely stiff, the width of the respective distance distribution can be accounted to the flexibility of the spin-label end group. It can be described by a Gaussian broadening ${ }^{16,17}$ with $\sigma=0.06 \mathrm{~nm}$. For all further experiments, we used this Gaussian broadening to take the flexibility of the spin-label end groups into account. The persistence length $\mathrm{L}_{\mathrm{p}}$ is a global parameter for describing the set of DEER data, the contour length $\mathrm{L}_{\mathrm{c}}$ was fitted for each oligomer individually. The obtained distance distributions are shown in Figure 2B, the fit parameters are listed in Table 1.

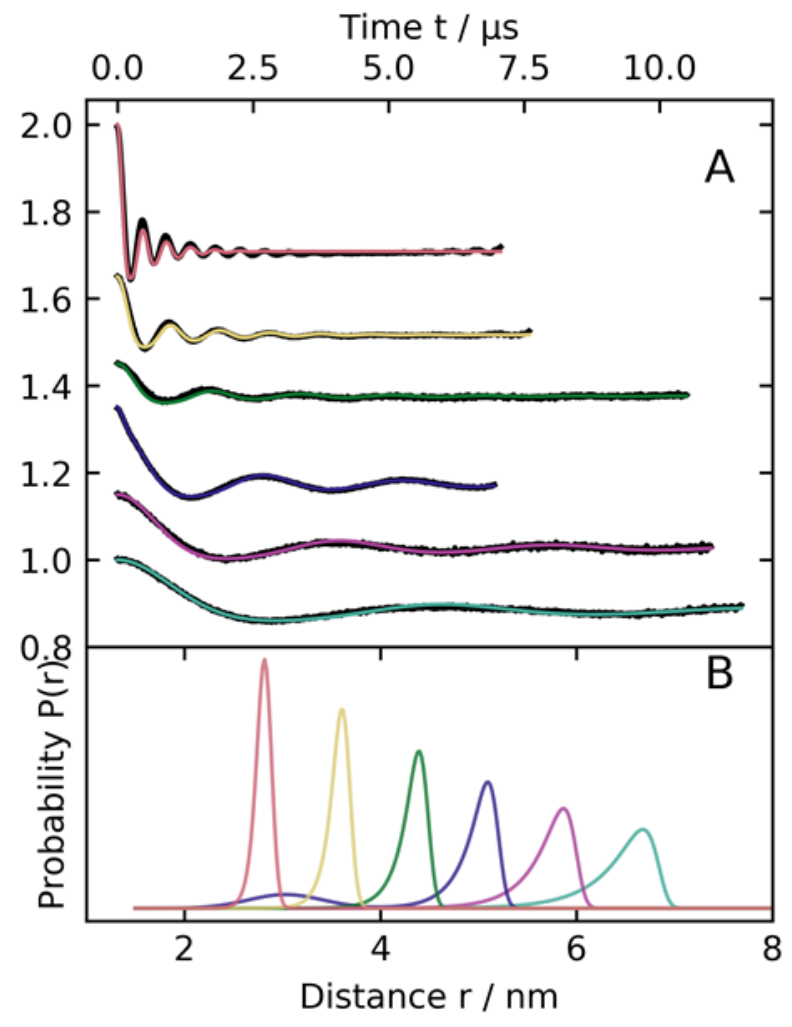

Figure 2. Distance measurement by DEER spectroscopy for doubly TEMPO-labeled reference oligomers with $\mathrm{n}$ from 1 (top) to 6 (bottom), that is $\mathbf{P}_{\mathbf{1}}, \mathbf{P}_{\mathbf{2}}, \mathbf{P}_{\mathbf{3}}, \mathbf{P}_{\mathbf{4}}, \mathbf{P}_{\mathbf{5}}{ }^{\prime}$ and $\mathbf{P}_{\mathbf{6}}{ }^{\prime}$, in toluene-d8. (A) Background corrected form factor with model fit (B) Distance distributions obtained by model fit, normalized to end-to-end distance probability.

Table 1. Oligomers and parameters of model-based fit with WLC. Global parameters: $\mathrm{L}_{\mathrm{p}}=14 \mathrm{~nm}$ and $\sigma=0.06 \mathrm{~nm}$. For $\mathrm{P}_{4}$ an additional gauss distance was introduced $(\mathrm{r}=3.03 \mathrm{~nm}, \sigma=0.267 \mathrm{~nm}$, $21 \%$ weighting factor).

\begin{tabular}{c|c} 
Oligomer & $\mathbf{L c}_{\mathbf{c}}[\mathbf{n m}]$ \\
\hline $\mathrm{P}_{1}$ & 2.90 \\
\hline $\mathrm{P}_{2}$ & 3.73 \\
\hline $\mathrm{P}_{3}$ & 4.56 \\
\hline $\mathrm{P}_{4}$ & 5.31 \\
\hline $\mathrm{P}_{5}^{\prime}$ & 6.14 \\
\hline $\mathrm{P}_{6}^{\prime}$ & 7.02
\end{tabular}


As an important detail, for $\mathbf{P}_{\mathbf{4}}$ we observed occurrence of three-fold bromination $(21 \%)$ in the course of the stepwise oligomer synthesis (see SI Figure S4.2) and attachment of a third spin-label in the center of the chain. This results in a broad additional distance contribution below the expected end-to-end distance, which is described by an additional Gaussian in the model (Table 1).

The set of doubly labeled oligomers can be described with $\mathrm{L}_{\mathrm{p}}=14 \mathrm{~nm}$ and $\mathrm{L}_{\mathrm{c}}$ is approx. $(2.1+\mathrm{n} \cdot 0.8) \mathrm{nm}$. This is in good agreement, with a monomer length of $0.75 \mathrm{~nm}$, found for poly(9,9-bis(2-ethylhexyl)fluorene-2,7-diyl) (PF2/6). ${ }^{27}$

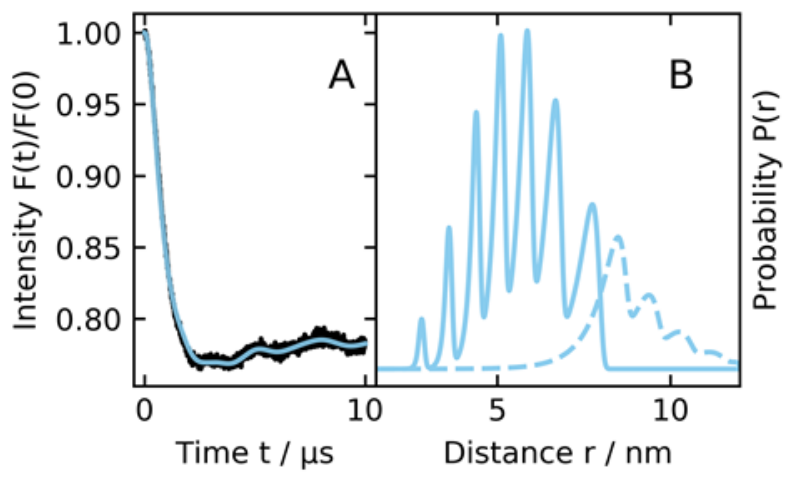

Figure 3. Distance measurement of polymer mixture from controlled Suzuki-Miyaura polymerization in toluene-d8. (A) Background corrected form factor with model fit (blue). (B) Distance distributions according to the fitted model taking a Poisson distribution for $\mathrm{n}=1-7$ (blue, solid) and for $\mathrm{n}>7$ (blue, dashed) with $\lambda=5.4$ with fixed global persistence length $\mathrm{L}_{\mathrm{p}}=14 \mathrm{~nm}$ into account.

With the WLC parameters for every oligomer in hand, DEER was applied to an oligomer mixture (Mix) obtained from controlled Suzuki-Miyaura polymerization (Figure 3A). A monomer to initiator species of five had been applied in the polymerization experiment.

The accessible range in the distance distribution detected by DEER is restricted by the dipolar evolution time, which was limited to $10 \mu \mathrm{s}$ in our experiments due to spin-spin relaxation. Under these conditions, distance contributions and their width can be detected with an upper limit of $7 \mathrm{~nm}$. The limit for accurate determination of the shape of the distribution is $5 \mathrm{~nm} .{ }^{4}$ To describe the experimental data, we used a model containing a superposition of different oligomers each described by WLC using the parameters obtained for the respected monodisperse reference oligomers $\left(\mathbf{P}_{\mathbf{1 - 4}}, \mathbf{P}_{\mathbf{5}}{ }^{\prime}, \mathbf{P}_{\mathbf{6}}{ }^{\prime}\right)$ according to a Poisson distribution with expectation value $\lambda$. As parameters for reference oligomers for $n>6$ were not derived individually, we fitted the contour length of an oligomer with $\mathrm{n}=7$ as an additional parameter in the analysis.

Note that the polymerization mixture contains oligomers with $n>7$, which are beyond the detection limit in our experiment. This does not disturb the analysis of the data for the oligomers amenable to DEER observation, however. To illustrate the amount of higher oligomers not observed by
DEER in the overall distributions, these were calculated according to the found Poisson distribution (Figure 3B, dotted line).

In summary, we find that the experimental DEER data is in excellent agreement with i) a Poisson distribution for the chain lengths, with an expectation value $\lambda=5.4$ and ii) a chain conformation described by a WLC with a persistence length of $14 \mathrm{~nm}$. Even when determining the fractions for each chain length individually in a model free approach, we find a reasonable agreement with a Poisson distribution (see SI S9). The agreement of the experimental DEER data with a Poisson distribution also allow for additional mechanistic conclusions on the polymerization reaction. Initiation of chains occurs very efficiently. That is, the initiating Pd-species is formed rapidly and completely in the early stages of the reaction, and it starts growth of a chain efficiently.

In many instances, solid polyfluorene materials are of interest rather than solutions. An access to chain conformations and chain flexibility in e.g. films or nanoparticles are desirable as they are instrumental in determining, for example, particle shapes. To demonstrate the principal suitability of the method reported also for nanoparticles, we prepared spherical poly(fluorene) nanoparticles (NP) by emulsification. ${ }^{28-32}$ The bulk polymer was blended with $\mathbf{P}_{\mathbf{1}}, \mathbf{P}_{\mathbf{2}}$ and $\mathbf{P}_{\mathbf{3}}$ (4:3:3 doubly labeled species, $0.25 \mathrm{mM}$ overall spin concentration in particle) by dissolution in tetrahydrofuran and emulsified in an aqueous solution of sodium dodecyl sulfate $(1.5 \mathrm{wt} \%)$ as stabilizing agent by ultrasonication. TEM and DLS measurements of the resulting stable dispersion confirmed the successful fabrication of spherical particles with an average diameter of approximately $190 \mathrm{~nm}$ (Figure 4A and B, see SI S6 for further characterization).

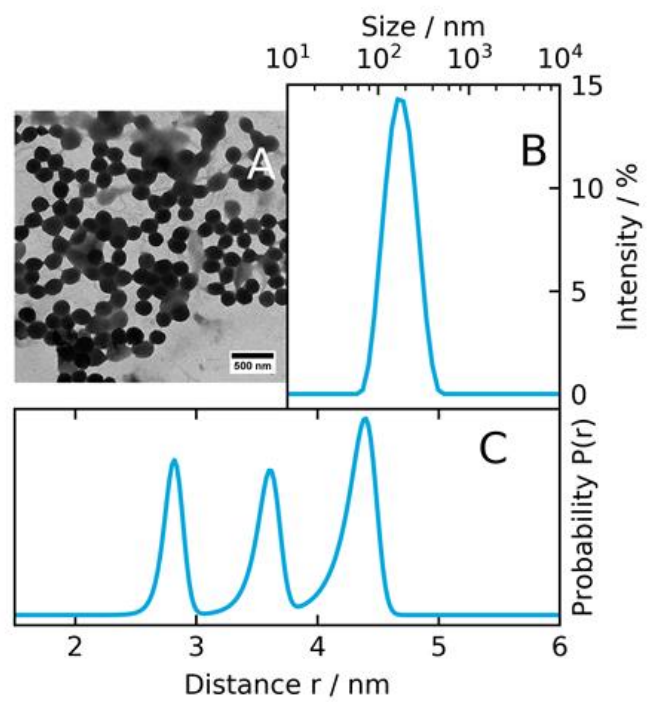

Figure 4. Poly(fluorene) particles with incorporated spin-labeled reference oligomers $\mathbf{P}_{\mathbf{1}}, \mathbf{P}_{\mathbf{2}}$ and $\mathbf{P}_{\mathbf{3}}$. (A) TEM image. (B) Particle size distribution measured by DLS. (C) Distance distributions obtained model fitting with three WLC and DEERNet background. Fit was performed with fixed persistence length $\left(\mathrm{L}_{\mathrm{p}}=14 \mathrm{~nm}\right)$, and fixed Gaussian broadening $\sigma=0.06 \mathrm{~nm}$. Weights from fit are $\mathrm{w}(\mathrm{n} 1)=0.32, \mathrm{w}(\mathrm{n} 2)=0.28, \mathrm{w}(\mathrm{n} 3)=0.40$. 
Prior to DEER measurements, the particles were purified by dialysis for the removal of excessive surfactant and freeze-dried. DEER measurements within the nanoparticle were performed with a dipolar evolution time of $3 \mu$ s due to increased spin-spin relaxation in the protonated environment of the nanoparticle. $\mathrm{T}_{\mathrm{m}}=1.9 \mu$ s compared to $8.0 \mu \mathrm{s}$ in the deuterated toluene-d8 matrix. The experimental data can be described by using contour length and broadening due to flexibility of the spin-label end group as derived in solution and fitting the weights for $\mathbf{P}_{\mathbf{1}}, \mathbf{P}_{\mathbf{2}}$ and $\mathbf{P}_{\mathbf{3}}$ as well as the global persistence length for the oligomers. Figure $4 \mathrm{c}$ shows the corresponding distance distribution for spin-labeled oligomers incorporated in nanoparticles. The found fractions of $32 \%, 28 \%$ and $40 \%$ are in agreement with the expectations from sample preparation (see above). We derived a persistence length of $\mathrm{L}_{\mathrm{p}}=14 \mathrm{~nm}$ as found in solution. This suggests a worm-like chain nature with undisturbed flexibility being retained in the nanoparticles.

\section{Conclusion}

In conclusion, we have demonstrated the application of DEER distance measurements to a real-life synthetic polymer containing a multitude of different chain length species. The required spin-labeled chain ends could be introduced efficiently by controlled Suzuki-Miyaura coupling Polymerization (sSMCP), cSMCP in general being a state-of-the-art versatile protocol for the synthesis of numerous poly(arylene)s. The DEER data agrees with a Poisson distribution, expected for the case of a living polymerization with fast and efficient initiation, which is given here. The method allows for the quantification of the individual n-mer populations and their respective conformations and flexibilities directly on mixtures. Further, we demonstrated that DEER allows a quantitative analysis of the oligomer fractions as well as characterization of conformation and flexibility inside nanoparticles in principle.

The necessity of spin-labeled oligomers is clearly a limitation of the analysis method reported. Thus, it is rather complementary than competitive to established standard methods for chain length distribution and conformation analysis. Its strength is access to properties of the individual n-mers directly on mixtures.

\section{ASSOCIATED CONTENT}

Supporting Information. Experimental procedures and characterization data; EPR measurements; Evaluation of DEER experiments.

\section{AUTHOR INFORMATION}

\section{Corresponding Author}

*E-mail: Stefan.Mecking@uni-konstanz.de

*E-mail: $\underline{\text { Malte.Drescher@uni-konstanz.de }}$

\section{Author Contributions}

The manuscript was written through contributions of all authors. All authors have given approval to the final version of the manuscript. $₫$ These authors contributed equally.
Notes

The authors declare no competing of interests.

\section{ACKNOWLEDGMENT}

Financial support by the DFG (SFB 1214, project A09) is gratefully acknowledged. We thank Silke Müller for MALDITOF measurements and Lars Bolk for support with GPC measurements.

\section{REFERENCES}

(1) Kuehne, A. J.C.; Gather, M. C.; Sprakel, J. Monodisperse Conjugated Polymer Particles by Suzuki-Miyaura Dispersion Polymerization. Nat. Commun. 2012, 3, 3:1088.

(2) Ciftci, S.; Kuehne, A. J. C. Monodisperse Conjugated Polymer Particles via Heck Coupling - A Kinetic Study to Unravel Particle Formation in Step-Growth Dispersion Polymerization. Macromolecules 2015, 48, 8389-8393.

(3) Pannier, M.; Veit, S.; Godt, A.; Jeschke, G.; Spiess, H.W. DeadTime Free Measurement of Dipole-Dipole Interactions between Electron Spins. J. Magn. Reson. 2000, 142, 331-340.

(4) Jeschke, G. DEER Distance Measurements on Proteins. Annu. Rev. Phys. Chem. 2012, 63, 419-446.

(5) Ruiz Perez, J. D.; Mecking, S. Anisotropic Polymer Nanoparticles with Tunable Emission Wavelengths by Intersegmental Chain Packing. Angew. Chem. Int. Ed. 2017, 56, 6147-6151.

(6) Leclerc, M.; Morin, J.-F. Design and Synthesis of Conjugated Polymers; John Wiley \& Sons, 2010.

(7) Yokoyama, A.; Suzuki, H.; Kubota, Y.; Ohuchi, K.; Higashimura, H.; Yokozawa, T. Chain-Growth Polymerization for the Synthesis of Polyfluorene via Suzuki-Miyaura Coupling Reaction from an Externally Added Initiator Unit. J. Am. Chem. Soc. 2007, 129, $7236-7237$.

(8) Burroughes, J. H.; Bradley, D. D. C.; Brown, A. R.; Marks, R. N.; Mackay, K.; Friend, R. H.; Burns, P. L.; Holmes, A. B. LightEmitting Diodes Based on Conjugated Polymers. Nature 1990, 347, 539-541.

(9) Friend, R. H.; Gymer, R. W.; Holmes, A. B.; Burroughes, J. H.; Marks, R. N.; Taliani, C.; Bradley, D. D.C.; Dos Santos, D. A.; Bredas, J. L.; Lögdlund, M. Electroluminescence in Conjugated Polymers. Nature 1999, 397, 121.

(10) Fischer, C. S.; Jenewein, C.; Mecking, S. Conjugated Star Polymers from Multidirectional Suzuki-Miyaura Polymerization for Live Cell Imaging. Macromolecules 2015, 48, 483-491.

(11) Elmalem, E.; Biedermann, F.; Johnson, K.; Friend, R. H.; Huck, W. T. S. Synthesis and Photophysics of Fully $\pi$-Conjugated Heterobis-Functionalized Polymeric Molecular Wires via Suzuki Chain-Growth Polymerization. J. Am. Chem. Soc. 2012, 134, 17769-17777.

(12) Zhang, H.-H.; Peng, W.; Dong, J.; Hu, Q.-S. $t$-Bu 3 P-Coordinated 2-Phenylaniline-Based Palladacycle Complex/ArBr as Robust Initiators for Controlled $\mathrm{Pd}(0) / t-\mathrm{Bu}_{3} \mathrm{P}$-Catalyzed Suzuki Cross-Coupling Polymerization of AB-Type Monomers. ACS Macro Lett. 2016, 5, 656-660.

(13) Yokozawa, T.; Suzuki, R.; Nojima, M.; Ohta, Y.; Yokoyama, A. Precision Synthesis of Poly(3-hexylthiophene) from CatalystTransfer Suzuki-Miyaura Coupling Polymerization. Macromol. Rapid Commun. 2011, 32, 801-806.

(14) Hubbell, W. L.; Mchaourab, H. S.; Altenbach, C.; Lietzow, M. A. Watching Proteins Move Using Site-Directed Spin Labeling. Structure 1996, 4, 779-783.

(15) Hubbell, W. L.; Altenbach, C. Investigation of Structure and Dynamics in Membrane Proteins Using Site-Directed Spin Labeling. Curr. Opin. Struct. Biol. 1994, 4, 566-573. 
(16) Hintze, C.; Schütze, F.; Drescher, M.; Mecking, S. Probing of Chain Conformations in Conjugated Polymer Nanoparticles by Electron Spin Resonance Spectroscopy. Phys. Chem. Chem. Phys. 2015, 17, 32289-32296.

(17) Godt, A.; Schulte, M.; Zimmermann, H.; Jeschke, G. How Flexible Are Poly(para-phenyleneethynylene)s? Angew. Chem. Int. Ed. 2006, 45, 7560-7564.

(18) Seo, K.-B.; Lee, I.-H.; Lee, J.; Choi, I.; Choi, T.-L. A Rational Design of Highly Controlled Suzuki-Miyaura Catalyst-Transfer Polycondensation for Precision Synthesis of Polythiophenes and Their Block Copolymers: Marriage of Palladacycle Precatalysts with MIDA-Boronates. J. Am. Chem. Soc. 2018, 140, 4335-4343. (19) Shiraki, T.; Shindome, S.; Toshimitsu, F.; Fujigaya, T.; Nakashima, N. Strong Main-Chain Length-Dependence for the $\beta$ Phase Formation of Oligofluorenes. Polym. Chem. 2015, 6, 51035109.

(20) Li, Y.; Ding, J.; Day, M.; Tao, Y.; Lu, J.; D'iorio, M. Synthesis and Properties of Random and Alternating Fluorene/Carbazole Copolymers for Use in Blue Light-Emitting Devices. Chem. Mater. 2004, 16, 2165-2173.

(21) Yeo, H.; Tanaka, K.; Chujo, Y. Tunable Optical Property between Pure Red Luminescence and Dual Emission Depended on the Length of Light-Harvesting Antennae in the Dyads Containing the Cardo Structure of BODIPY and Oligofluorene. Macromolecules 2016, 49, 8899-8904.

(22) Kratky, O.; Porod, G. Röntgenuntersuchung Gelöster Fadenmoleküle. Recl. Trav. Chim. Pays-Bas 1949, 68, 1106-1122.

(23) Wilhelm, J.; Frey, E. Radial Distribution Function of Semiflexible Polymers. Phys. Rev. Lett. 1996, 77, 2581.

(24) Jeschke, G.; Chechik, V.; Ionita, P.; Godt, A.; Zimmermann, H.; Banham, J.; Timmel, C. R.; Hilger, D.; Jung, H. DeerAnalysis2006 - A Comprehensive Software Package for Analyzing Pulsed ELDOR Data. Appl. Magn. Reson. 2006, 30, 473-498.

(25) Edwards, T. H.; Stoll, S. Optimal Tikhonov Regularization for DEER Spectroscopy. J. Magn. Reson. 2018, 288, 58-68.

(26) Worswick, S. G.; Spencer, J. A.; Jeschke, G.; Kuprov, I. Deep Neural Network Processing of DEER Data. Sci. Adv. 2018, eaat5218.

(27) Fytas, G.; Nothofer, H. G.; Scherf, U.; Vlassopoulos, D.; Meier, G. Structure and Dynamics of Nondilute Polyfluorene Solutions. Macromolecules 2002, 35, 481-488.

(28) Pecher, J.; Mecking, S. Nanoparticles of Conjugated Polymers. Chem. Rev. 2010, 110, 6260-6279.

(29) Wu, C.; McNeill, J. Swelling-Controlled Polymer Phase and Fluorescence Properties of Polyfluorene Nanoparticles. Langmuir 2008, 24, 5855-5861.

(30) Wu, C.; Szymanski, C.; McNeill, J. Preparation and Encapsulation of Highly Fluorescent Conjugated Polymer Nanoparticles. Langmuir 2006, 22, 2956-2960.

(31) Landfester, K.; Montenegro, R.; Scherf, U.; Güntner, R.; Asawapirom, U.; Patil, S.; Neher, D.; Kietzke, T. Semiconducting Polymer Nanospheres in Aqueous Dispersion Prepared by a Miniemulsion Process. Adv. Mater. 2002, 14, 651-655.

(32) Kietzke, T.; Neher, D.; Kumke, M.; Montenegro, R.; Landfester, K.; Scherf, U. A Nanoparticle Approach to Control the Phase Separation in Polyfluorene Photovoltaic Devices. Macromolecules 2004, 37, 4882-4890. 